\title{
Differences in volumetric bone mineral density (vBMD) at the radius and tibia in premenopausal Caucasian and Asian women
}

\author{
O. Hakim ${ }^{1}$, A. Darling ${ }^{1}$, K. Hart ${ }^{1}$, J. Berry ${ }^{2}$ and S. Lanham-New ${ }^{1}$.
}

${ }^{1}$ Nutritional Sciences Division, Faculty of Health and Medical Sciences, University of Surrey, Guildford; ${ }^{2}$ Vitamin D Research Group, Department of Medicine, University of Manchester.

Current studies indicate that women of European origin have higher bone mineral density (BMD) than Far-East Asian women but few data exist on South Asian women. Roy et al (2005) explained differences in BMD between Caucasian and Asian women by differences in bone size ${ }^{1}$. The aim of this study was to investigate differences between volumetric bone mineral density (vBMD) between Caucasian (C) and Asian (A) women. Thirty-five healthy premenopausal women (19 C and 16 A), age range 18-55 yrs, were studied. Peripheral Quantitative Computed Tomography (pQCT) measurements were taken at the radius and tibia (non-dominant) using a Stratec XCT 2000 pQCT scanner.

Caucasian were slightly lighter and taller than Asian women, with an average BMI of 24.9 (4.12) and $27.7(5.8)$ respectively. The table below shows the results of vBMD at $4 \%$ and $66 \%$ radius; $4 \%, 14 \%$, and $38 \%$ tibia in $\mathrm{C}$ and $\mathrm{A}$ women

\begin{tabular}{|c|c|c|c|}
\hline & & Caucasian $(n=22)$ & Asians $(n=19)$ \\
\hline \multirow{2}{*}{ 罟 } & TOT_DEN (mg/cm3) & $315.6(45.3)$ & 312.1(45.6) \\
\hline & TRAB_DEN (mg/cm3) & $178.9(35.5)$ & 175.3(37.2) \\
\hline \multirow{2}{*}{ 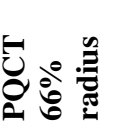 } & TOT_DEN (mg/cm3) & $746.6(86.3)$ & 768.8(85.9) \\
\hline & CORT_DEN (mg/cm3) & $1134.7(38.4)$ & $1126.3(42.5)$ \\
\hline \multirow{2}{*}{ 帠 } & TOT_DEN (mg/cm3) & $303.3(31.7)$ & $343.6(53.4)^{*}$ \\
\hline & TRAB_DEN (mg/cm3) & $228.7(29.2)$ & $272.4(66.9)^{*}$ \\
\hline \multirow{2}{*}{ 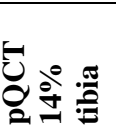 } & TOT_DEN (mg/cm3) & $571.6(117.4)$ & $495.5(92.1)^{*}$ \\
\hline & CORT_DEN (mg/cm3) & 1135.6(24.4) & 1095.0(65.5)* \\
\hline \multirow[b]{2}{*}{ 舁罟 } & TOT_DEN (mg/cm3) & 903.6(76.9) & 856.8(85.2) \\
\hline & CORT_DEN (mg/cm3) & 1179.8(19.4) & $1160.9(53.3)$ \\
\hline
\end{tabular}

${ }^{*} \mathrm{p}<0.05,{ }^{* *} \mathrm{p}<0.01$ for 2 sample $t$-test. Data expressed as the mean (SD); Abbreviations: TOT_DEN, total density; TRAB_DEN, trabecular density; TOT_A, total area; TRAB_A, trabecular area; CORT_DEN, cortical density; CORT_A, cortical area.

Asian women had significantly higher total density and trabecular density at $4 \%$ tibia than C women; but they had significantly lower cortical density at $14 \%$ tibia site. Asian women had also significant lower total area and trabecular area $(p<0.05)$ at $4 \%$ tibia site. There were no significant differences in vBMD between $C$ and $A$ in radius sites. (Not shown in the table) however, at the $4 \%$ radius, Asian have significant lower bone mass $(p<0.05)$, total area $(p<0.01)$ and trabecular area $(p<0.01)$ than Caucasian. Our finding supports the results of Ward et al (2007) where there was no difference in vBMD at the radius between $\mathrm{A}$ and $\mathrm{C}$ women ${ }^{2}$. Our novel findings for differences in the tibia sites for $\mathrm{A}$ and $\mathrm{C}$ women require further investigations as there are no data examining this bone site in ethnic groups.

References:

${ }^{1}$ Roy et. al. Osteoporosis Int. 2007; 41: 117-121

${ }^{2}$ Ward et. al. Bone. 2005; 16; 1254-1262 